\title{
Influence of grain boundaries on the properties of polycrystalline germanium
}

Cite as: J. Appl. Phys. 128, 075301 (2020); https://doi.org/10.1063/5.0006469

Submitted: 02 March 2020 • Accepted: 16 July 2020 • Published Online: 17 August 2020

(D) T. Imajo, (D) T. Suemasu and (iD K. Toko
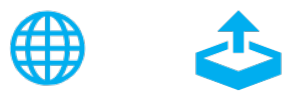

View Online

Export Citation

\section{ARTICLES YOU MAY BE INTERESTED IN}

$\mathrm{Zn}$-induced layer exchange of $\mathrm{p}$ - and $\mathrm{n}$-type nanocrystalline SiGe layers for flexible thermoelectrics

Applied Physics Letters 116, 182105 (2020); https://doi.org/10.1063/5.0006958

Polycrystalline thin-film transistors fabricated on high-mobility solid-phase-crystallized Ge on glass

Applied Physics Letters 114, 212107 (2019); https://doi.org/10.1063/1.5093952

Layer-exchange crystallization for low-temperature $\left(\sim 450^{\circ} \mathrm{C}\right)$ formation of $\mathrm{n}$-type tensilestrained Ge on insulator

Applied Physics Letters 117, 172102 (2020); https://doi.org/10.1063/5.0020489

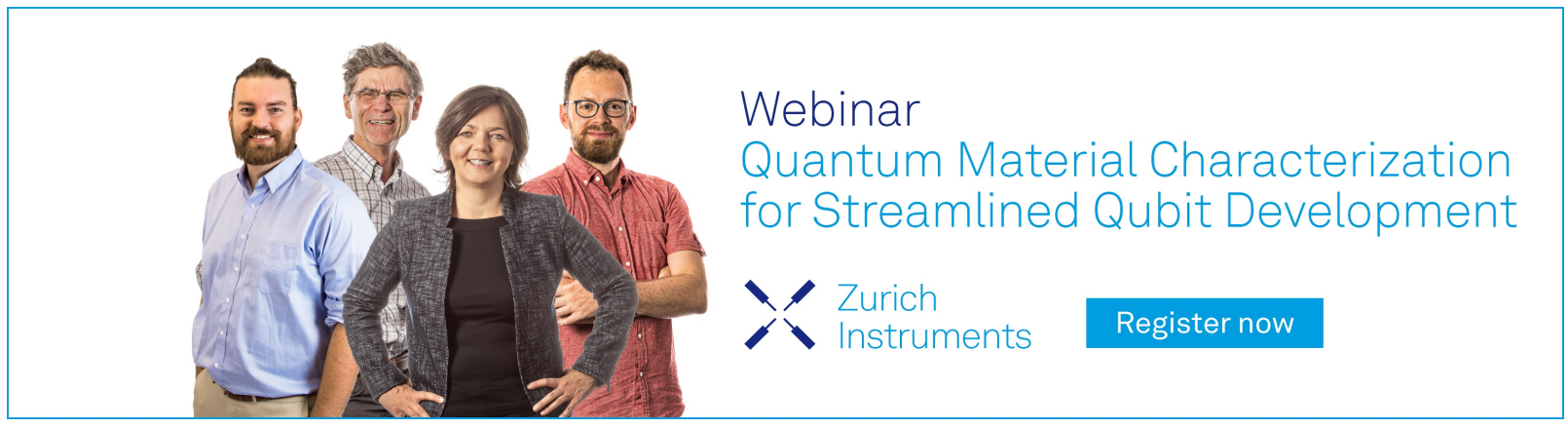




\title{
Influence of grain boundaries on the properties of polycrystalline germanium
}

\author{
Cite as: J. Appl. Phys. 128, 075301 (2020); doi: 10.1063/5.0006469 \\ Submitted: 2 March 2020 - Accepted: 16 July 2020 . \\ Published Online: 17 August 2020
}

T. Imajo, $^{1, a), b) ~(D) ~ T . ~ S u e m a s u, ~}{ }^{7}$ (D) and K. Toko ${ }^{1,2, b)}$ (D)

\author{
AFFILIATIONS \\ ${ }^{1}$ Institute of Applied Physics, University of Tsukuba, 1-7-1 Tennodai, Tsukuba, Ibaraki 305-8573, Japan \\ ${ }^{2}$ PRESTO, Japan Science and Technology Agency, 4-7-8 Honcho, Kawaguchi, Saitama 332-0012, Japan \\ a)JSPS Research Fellow, 8 Ichiban-cho, Chiyoda-ku, Tokyo 102-8472, Japan
}

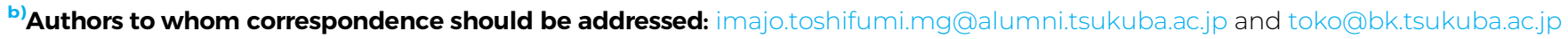

\begin{abstract}
High-speed thin film transistors based on plastic substrates are indispensable to realize next-generation flexible devices. Here, we synthesized a polycrystalline $\mathrm{Ge}$ layer, which had the highest quality ever, on $\mathrm{GeO}_{2}$-coated substrates using advanced solid-phase crystallization at $375^{\circ} \mathrm{C}$. X-ray diffraction and Raman spectroscopy revealed that Ge on plastic had a compressive strain, while conventional Ge with a glass substrate had a tensile strain. This behavior was explained quantitatively from the difference in the thermal expansion coefficients between Ge and the substrate. Electron backscatter diffraction analyses showed that the Ge had large grains up to $10 \mu \mathrm{m}$, while many intragranular grain boundaries were present. The potential barrier height of the grain boundary was lower for the plastic sample than that for the glass sample, which was discussed in terms of the strain direction. These features resulted in a hole mobility $\left(500 \mathrm{~cm}^{2} / \mathrm{V} \mathrm{s}\right)$ exceeding that of a single-crystal Si wafer. The findings and knowledge will contribute to the development of polycrystalline engineering and lead to advanced flexible electronics.
\end{abstract}

Published under license by AIP Publishing. https://doi.org/10.1063/5.0006469

\section{INTRODUCTION}

For the coming Internet-of-Things society, the development of flexible electronics based on plastic substrates is increasingly desired because they are lightweight, flexible, and economical. Fabricating high-speed thin film transistors (TFTs) on plastic substrates is the key to dramatically improve the functionality of flexible devices. Ge has been proposed as a major candidate for next-generation transistor channel materials due to its high carrier mobility and good compatibility with $\mathrm{Si}^{1-3}$ The field-effect mobility of $\mathrm{Ge}$ metal-oxide-semiconductor field-effect transistors (MOSFETs) has surpassed that of Si MOSFETs due to the developments of device technologies, including gate stacks. ${ }^{4-6}$ However, for bulk-Ge, there is a problem with the leak current caused by the narrow bandgap $(0.66 \mathrm{eV})$. Fabricating $\mathrm{Ge}$ on an insulator (GOI) structure is the most promising approach to reduce leakage current. ${ }^{6}$ The GOI structure has been obtained using mechanical transfer, ${ }^{7-9}$ oxidation-induced condensation, ${ }^{5,10}$ rapid-melting growth, ${ }^{11-14}$ lamp annealing, ${ }^{15,16}$ and epitaxial growth on Si-on-insulator. ${ }^{17}$ Although the resulting Ge layers have a high crystallinity, these methods require single-crystal substrates, complicated processes, or high temperatures, so that applications to low-heat-resistant plastic substrates are difficult. Essentially, the crystallization temperature of $\mathrm{Ge}$ is relatively low for an inorganic semiconductor. Taking advantage of this feature, many techniques to synthesize polycrystalline (poly-) $\mathrm{Ge}$ at low temperatures on glass and plastic substrates have been studied. ${ }^{18-28}$ Ge-TFTs have been established using poly-Ge formed by solid-phase crystallization (SPC), ${ }^{29-31}$ laser annealing, ${ }^{32}$ seed layer technique, $^{33}$ and metal-induced crystallization. ${ }^{34-37}$ However, poly-Ge generally has a high hole concentration due to defect-induced acceptors and a low hole mobility due to grain boundary (GB) scattering, ${ }^{18,38}$ limiting the TFT performance.

In the last few years, we have developed an advanced SPC technique using a densified amorphous (a-) Ge precursor and updated the Hall hole mobility of poly-Ge to $340 \mathrm{~cm}^{2} / \mathrm{V} \mathrm{s}^{39}$ and the electron mobility to $370 \mathrm{~cm}^{2} / \mathrm{V} \mathrm{s}{ }^{40,41}$ Accumulation-mode p-channel TFTs based on the SPC-Ge exhibited the highest field-effect mobility $\left(170 \mathrm{~cm}^{2} / \mathrm{V} \mathrm{s}\right)$ among low-temperature $\left(<500^{\circ} \mathrm{C}\right)$ poly-Ge-TFTs without minimizing the channel region $(<1 \mu \mathrm{m}){ }^{42}$ We further updated the Hall hole mobility to $620 \mathrm{~cm}^{2} / \mathrm{V} \mathrm{s}$ by thickening $\mathrm{Ge}$, postannealing at $500{ }^{\circ} \mathrm{C}$, and inserting a $\mathrm{GeO}_{2}$ underlayer. ${ }^{43,44}$ 
This hole mobility is the highest ever recorded for a semiconductor film directly grown on an insulator at temperatures below $900^{\circ} \mathrm{C}$.

In the present study, we deploy SPC-Ge on a flexible plastic substrate and evaluated the crystallinity and electrical properties, while comparing Ge on a glass substrate. The residual strain in the SPC-Ge is compressive on plastic but tensile on glass, affecting the grain boundary properties.

\section{EXPERIMENT}

We prepared a 50-nm-thick $\mathrm{GeO}_{2}$ layer on $\mathrm{SiO}_{2}$ glass and plastic (125- $\mu \mathrm{m}$-thick polyimide: Kapton) substrates using RF magnetron sputtering (base pressure $3.0 \times 10^{-4} \mathrm{~Pa}$ ) with an RF power of $50 \mathrm{~W}$. After that, a-Ge precursors were prepared using a Knudsen cell of a molecular beam deposition system (base pressure: $5 \times 10^{-7} \mathrm{~Pa}$ ) while heating the samples at $150{ }^{\circ} \mathrm{C}$ to densify the a-Ge. ${ }^{39}$ The thickness of the a-Ge layer, $t$, ranged from 100 to $500 \mathrm{~nm}$. The Ge sources (Furuuchi Chemical Corporation) had a purity of $99.999 \%$. The samples were then loaded into a conventional tube furnace in a $\mathrm{N}_{2}(99.9 \%)$ atmosphere and annealed at $375^{\circ} \mathrm{C}$ for $150 \mathrm{~h}$ to induce SPC. We note that the lower annealing temperature produces the larger crystal grains; however, this requires a longer annealing time. ${ }^{39,41}$ To visualize the grain boundaries clearly, we selected the slow annealing conditions that maximize the grain size. The crystal quality of the grown Ge layers was evaluated using x-ray diffraction (XRD; Rigaku SmartLab) with $\mathrm{Cu} \mathrm{K} \alpha$ radiation, Raman spectroscopy (JASCO NRS-5100, spot diameter $20 \mu \mathrm{m}$, wavelength $532 \mathrm{~nm}$ ), scanning electron microscopy (SEM; JEOL JSM-7001F), and electron backscatter diffraction (EBSD; TSL OIM analysis). The electrical properties of the SPC-Ge layers were evaluated using Hall effect measurements (Bio-Rad HL5500PC).

\section{RESULTS AND DISCUSSION}

Figure 1 (a) shows the $\theta-2 \theta$ XRD patterns corresponding to poly-Ge for the samples on glass and plastic substrates. For both glass and plastic samples, the intensity ratio of each peak almost agrees with the reference data from Ge powder diffraction patterns (JCPDS 04-0545), indicating that the Ge layers are randomly oriented. The inset shows that the Ge 111 peak of each SPC-Ge shifts in the opposite direction from that of a bulk-Ge (111) wafer. Figure 1(b) shows the position $(2 \theta)$ of the Ge 111 peak derived for $t=300-500 \mathrm{~nm}$. We note that the samples with $t \leq 200 \mathrm{~nm}$ are too thin to obtain enough peak intensity to recognize. For all $t$ (300$500 \mathrm{~nm}$ ), the Ge 111 peak in the glass samples shifts to higher degrees, while that in the plastic samples shifts to lower degrees compared with that of bulk-Ge. These results suggest that, in the perpendicular direction, the Ge layer has compressive strain for glass samples and tensile strain for plastic samples.

The Raman spectra in Fig. 2(a) show the broad peaks corresponding to a-Ge before annealing and the sharp peaks corresponding to crystalline $\mathrm{Ge}(\mathrm{c}-\mathrm{Ge})$ after annealing. This behavior is observed for all $t(100-500 \mathrm{~nm})$ for both glass and plastic samples. The wavenumber of the c-Ge peak of each SPC-Ge shifts in the opposite direction from that of bulk-Ge. Figure 2(b) shows that, for all $t$, the $\mathrm{c}-\mathrm{Ge}$ peak in glass samples shifts to a lower wavenumber, while that in plastic samples shifts to a higher wavenumber from that of bulk-Ge. These results are consistent with the XRD results

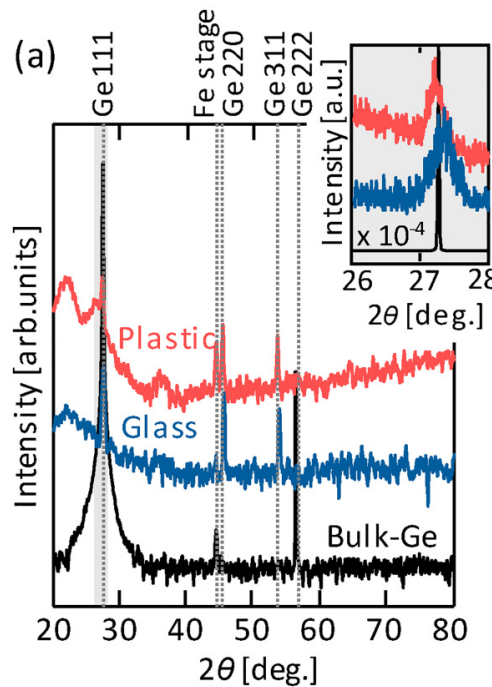

(b)

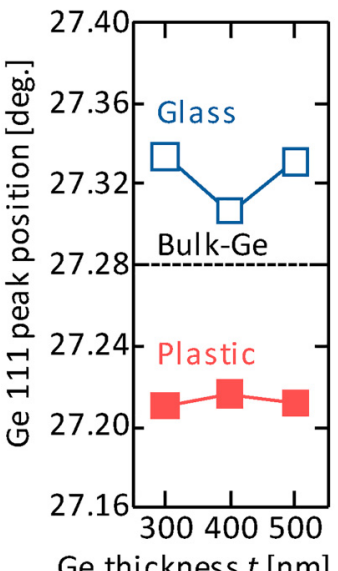

FIG. 1. XRD study of the SPC-Ge layers on glass and plastic substrates. (a) $\theta-2 \theta$ XRD patterns for $t=500 \mathrm{~nm}$, where the data for a bulk-Ge (111) wafer is shown for comparison. The inset is a magnified view focusing on the Ge 111 peaks. (b) Ge 111 peak position $(2 \theta)$ as a function of $t$, where the value for a bulk-Ge (111) wafer is shown by the dotted line.

(Fig. 1) because in the in-plane direction, a low wavenumber shift corresponds to tensile strain, while a high wavenumber shift corresponds to compressive strain. ${ }^{45}$ According to the Raman shifts and the equation proposed by Chen et al., ${ }^{45}$ the tensile strain in the glass sample is $\sim 0.48 \%$ and the compressive strain in the plastic sample is $\sim 0.70 \%$. According to Hooke's law and Young's modulus of $\mathrm{Ge}$, the stress on the Ge layer is estimated to be $0.49 \mathrm{GPa}$ on glass and $0.72 \mathrm{GPa}$ on plastic, respectively. The cause of the strains can be explained from the perspective of the thermal expansion difference between the substrate and Ge. ${ }^{46,47}$ The thermal expansion coefficients of $\mathrm{Ge}, \mathrm{SiO}_{2}$ glass, and the current polyimide plastic are $5.8 \times 10^{-6} \mathrm{~K}^{-1}, 0.5 \times 10^{-6} \mathrm{~K}^{-1}$, and $27 \times 10^{-6} \mathrm{~K}^{-1}$, respectively. Considering the relationship of these thermal expansion coefficients, tensile strain is applied to $\mathrm{Ge}$ on glass while compressive strain is applied to Ge on plastic during the cooling process after Ge crystallization. The strain amount $\varepsilon_{\text {th }}$ in a thin film caused by the thermal expansion difference is expressed by the following equation: ${ }^{48}$

$$
\varepsilon_{\mathrm{th}}=\left(\alpha_{\mathrm{f}}-\alpha_{\mathrm{s}}\right) \frac{\Delta T}{1-v},
$$

where $\alpha_{\mathrm{f}}$ and $\alpha_{\mathrm{s}}$ are the thermal expansion coefficients of the thin film and the substrate, respectively, $\Delta T$ is the temperature causing thermal expansion, and $v$ is Poisson's ratio of the thin film. According to Eq. (1), when the sample is cooled from the annealing temperature of $375^{\circ} \mathrm{C}$ to room temperature (RT), the tensile strain of $\mathrm{Ge}$ on glass is $0.25 \%$ and the compressive strain of $\mathrm{Ge}$ on plastic is $1.02 \%$. These are on the same order as the strain estimated from the Raman shift. Figure 2(c) shows that the full width 

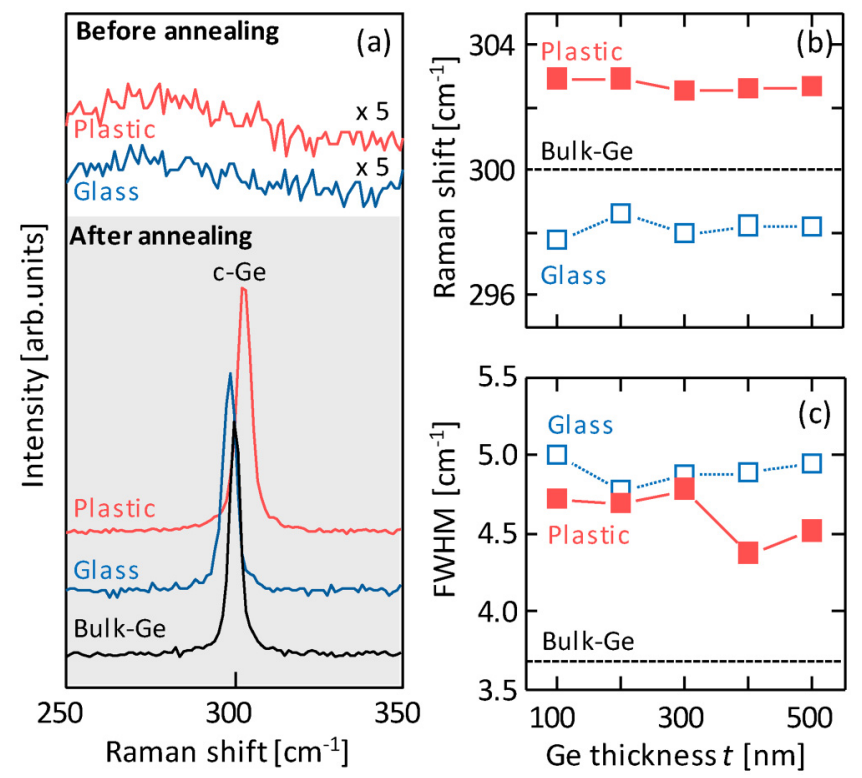

FIG. 2. Raman spectroscopy study of the SPC-Ge layers on glass and plastic substrates. (a) Raman spectra of the samples before and after annealing for $t=500 \mathrm{~nm}$, where the spectra for a bulk-Ge wafer are shown for comparison. (b) Raman shifts and (c) FWHMs of the c-Ge peaks as a function of $t$, where the values for a bulk-Ge wafer are shown by the dotted lines. (a)
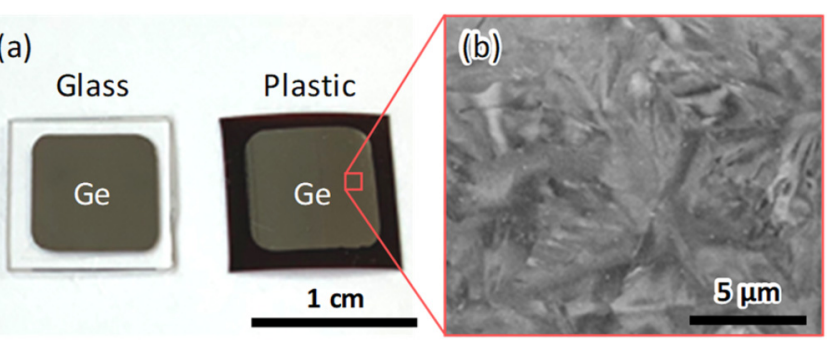

(c)

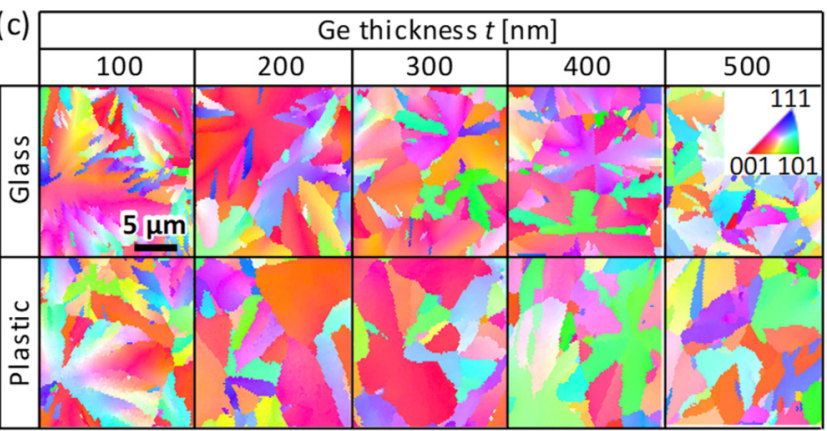

FIG. 3. (a) Photograph of the SPC-Ge samples. (b) Secondary electron image obtained from SEM (acceleration voltage: $25 \mathrm{kV}$ ) for the plastic sample for $t=100 \mathrm{~nm}$. (c) IPF images of the samples summarized as the matrix composed of the substrate material and $t$. Colors indicate the crystal orientation, according to the inserted color key. at half maximum (FWHM) of the c-Ge peak is almost constant with respect to $t$, while the plastic samples exhibit lower FWHMs than the glass samples for all $t$. This behavior indicates that the plastic samples have higher crystallinity than the glass samples.

Figure 3(a) shows a representative photograph of the SPC-Ge samples with glass and plastic substrates. The SEM image in Fig. 3(b) shows a contrast corresponding to the crystal orientation visualized by the electron channeling effect for high-quality crystals, which is not remarkable for low-quality polycrystalline films. ${ }^{39}$ The inverse pole figures (IPFs) in Fig. 3(c) show that the crystal orientation is almost random for all samples, which is consistent with the XRD results [Fig. 1(a)]. To investigate the distribution of GBs in detail, the IPF images are converted to grain maps, as shown in Fig. 4(a). A grain is defined as an area surrounded by GBs. Figure 4(a) shows that the SPC-Ge consists of grains on the $\mu \mathrm{m}$-order for all samples. Figure 4(b) shows that the average grain size, referred to as the area-weighted average, is almost the same for glass and plastic samples. The grain size exceeds $8 \mu \mathrm{m}$ for all samples except $t=500 \mathrm{~nm}$. The grain size reduction in large $t$ is likely because the nucleation in the bulk increases with increasing $t^{28,43}$ The density of GBs surrounding (i.e., identifying) grains shows a relatively high value at $t=500 \mathrm{~nm}$, which is consistent with the behavior of the grain size.

We analyzed intragranular GBs and classified them into lowangle $\left(<15^{\circ}\right) \mathrm{GB}(\mathrm{LAGB})$, high-angle $\left(\geq 15^{\circ}\right) \mathrm{GB}$ (HAGB), and twin boundaries. Figure 4(a) shows that intragranular GBs are present in most grains and extend radially. In addition, most HAGBs are connected to twin boundaries. Figures 4(c) shows that the density of twin boundaries becomes noticeable at large $t$, while Figs. 4(d) and 4 (e) show that the density of LAGBs and HAGBs become noticeable at small $t$. Figure 4(a) shows that the crystal rotation in grains develops LAGB and turns a twin boundary into HAGB during the lateral growth. According to Fig. 3(c), the crystal rotation becomes more pronounced in smaller $t$, which accounts for the results in Fig. 4. The mechanism leading to the crystal rotation remains unclear, but it may possibly be due to the film strain: the smaller $t$ provides the larger strains [Fig. 2(b)]. For most $t$, plastic samples have a lower density of intragranular GBs than glass samples, which accounts for the results of the Raman FWHM [Fig. 2(c)]. The crystallinity difference may be related to the difference in the thermal expansion strain during the SPC. For TFT fabrication, grain boundary control is desired for excellent operation. Because the Ge grain size is much larger than the channel region $(<\mu \mathrm{m})$, it will be possible to make the channel region into a single crystal if the starting point of nucleation can be controlled. Applying a metal catalyst or stress in a local area is a possible approach to control the nucleation and then grain boundaries. ${ }^{49,50}$

We evaluated the electrical properties of the samples. All samples show p-type conduction, similar to conventional undoped SPC-Ge. ${ }^{18}$ This is because the defects in Ge provide shallow acceptor levels, which generate holes at RT. ${ }^{38}$ As shown in Fig. 5(a), the hole concentration $p$ is almost constant with respect to $t$, while the hole mobility $\mu$ increases with increasing $t$. Both $p$ and $\mu$ have no significant differences between glass and plastic samples. The 


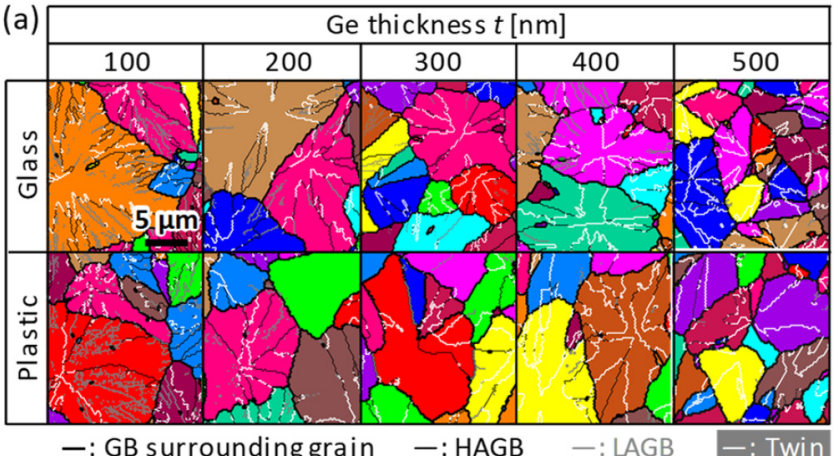

- : GB surrounding grain $\quad-$ : HAGB $\quad-$ : LAGB $\quad-$ : Twin
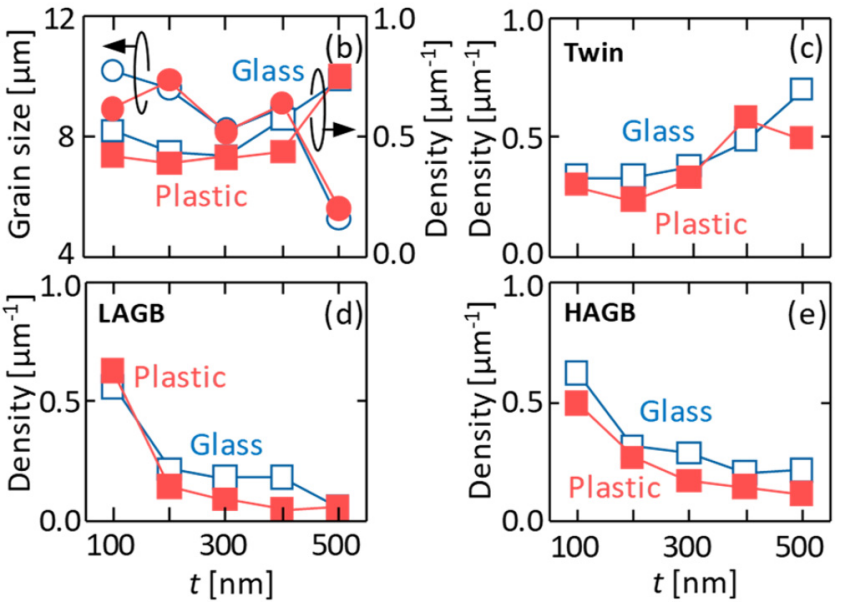

FIG. 4. EBSD analyses of the SPC-Ge layers on glass and plastic substrates. (a) Grain map of the samples summarized as the matrix composed of substrate material and $t$. Each colored area corresponds to one grain. Thick black lines show GBs surrounding the grains, thin black lines show HAGBs, gray lines show LAGBs, and white lines show twin boundaries. (b) Average grain size and the density of GBs surrounding the grains as a function of $t$. Intragranular density of (c) twin boundary, (d) LAGB, and (e) HAGB as a function of $t$.

highest $\mu$ reached $500 \mathrm{~cm}^{2} / \mathrm{V} \mathrm{s}$ for the plastic sample with $t=400 \mathrm{~nm}$. We estimated GB barrier height $E_{\mathrm{B}}$ by the conduction model in polycrystalline semiconductors proposed by Seto. ${ }^{51}$ According to this model, $\mu$ limited by GB scattering can be determined using the following equation:

$$
\mu=\frac{L q}{\sqrt{2 \pi m^{*} k T}} \exp \left(-\frac{E_{B}}{k T}\right),
$$

where $T$ is the absolute temperature, $L$ is the grain size, $m^{*}$ is the effective mass, and $k$ is the Boltzmann constant. As shown in the inset in Fig. 5(b), for $t=100 \mathrm{~nm}$, the Arrhenius plots of $\mu T^{1 / 2}$ exhibit a downward-sloping straight line, indicating that the carrier conduction in the poly-Ge layer is dominantly limited by GB scattering. ${ }^{51}$ Conversely, for $t=400 \mathrm{~nm}, \mu T^{1 / 2}$ increases with increasing $1000 / T$ and then begins to decrease. This behavior suggests that $\mu$ is limited by impurity scattering near RT but is limited by GB (a)

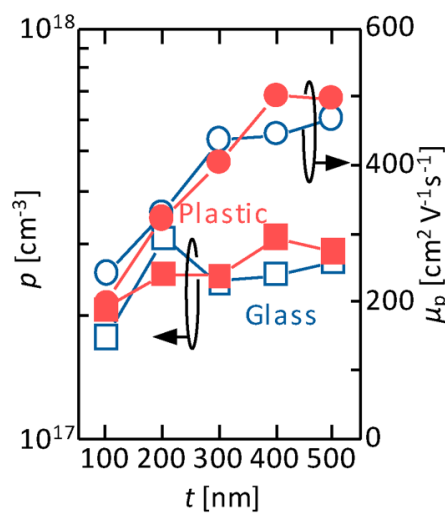

(b)

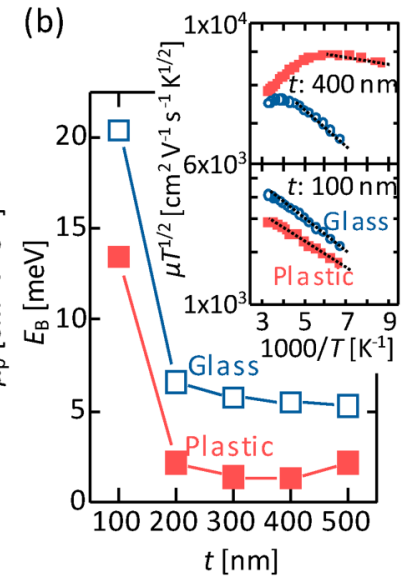

FIG. 5. Electrical properties of the SPC-Ge layers on glass and plastic substrates. (a) Hole concentration $p$ and hole mobility $\mu$ as a function of $t$. (b) GB barrier height $E_{\mathrm{B}}$ as a function of $t$. Inset shows representative Arrhenius plots of $\mu T^{1 / 2}$ for $t=100 \mathrm{~nm}$ and $400 \mathrm{~nm}$, where the dotted lines are the fitting results to derive $E_{\mathrm{B}}$.

scattering at low temperature. In addition, the shapes of the Arrhenius plots significantly differ between glass and plastic samples, indicating a large difference of $E_{\mathrm{B}}$. Figure 5(b) shows that $E_{\mathrm{B}}$ decreases with increasing $t$. Particularly, for $t=100 \mathrm{~nm}, E_{\mathrm{B}}$ shows high values over $20 \mathrm{meV}$ for the glass sample and $13 \mathrm{meV}$ for the plastic sample. These $E_{\mathrm{B}}$ values are much larger than that of SPC-Ge on $\mathrm{SiO}_{2}{ }^{39}$ and a-Si, ${ }^{28}$ suggesting that the GB property near the $\mathrm{Ge} / \mathrm{GeO}_{2}$ interface is inferior to those of $\mathrm{Ge} / \mathrm{SiO}_{2}$ and $\mathrm{Ge} / \mathrm{a}-\mathrm{Si}$. Therefore, for thin Ge layers $(t \leq 100 \mathrm{~nm})$, the a-Si underlayer might be more suitable than $\mathrm{GeO}_{2}$ for higher $\mu{ }^{28}$ We note that the plastic sample exhibits a lower $E_{\mathrm{B}}$ than that of the glass sample for all $t$. The minimum $E_{\mathrm{B}}$ reaches $1.3 \mathrm{meV}$ for the plastic sample with $t=400 \mathrm{~nm}$. The $E_{\mathrm{B}}$ difference is explained from the perspective of strain (Figs. 1 and 2). For the glass sample, Ge has in-plane tensile strain, which likely increases dangling bonds (i.e., trap density) in the GBs, increasing $E_{\mathrm{B}}$. In contrast, for the plastic sample, Ge has in-plane compressive strain, which promotes Ge bonding in the GBs, decreasing $E_{\mathrm{B}}$. Therefore, these results suggest that the strain direction in a poly-Ge layer affects its $E_{\mathrm{B}}$, which will be useful for engineering polycrystalline materials.

To investigate the mechanical strength of the Ge layers on the plastic substrate, we performed a simple tape test and bending test. As shown in Fig. 6(a), when the Kapton tape attached to the entire sample surface was peeled off, the Ge layer remained attached to the plastic substrate. This result indicates that the Ge layer is firmly adhered to the substrate. The samples for $t=100 \mathrm{~nm}$ and $400 \mathrm{~nm}$ were bent as representatively shown in Fig. 6(b) and then evaluated by Hall effect measurements. Figure 6(c) shows that the $\mu$ values are maintained up to a bend angle of $50^{\circ}$, while decreases significantly when the bend angle exceeds $\sim 50^{\circ}$. The decrease in $\mu$ is due to the occurrence of cracks in the film. Because the cracks may be suppressed when the Ge layer is isolated for TFTs, the bending cycle effects on the TFT performance should be investigated in the future. 

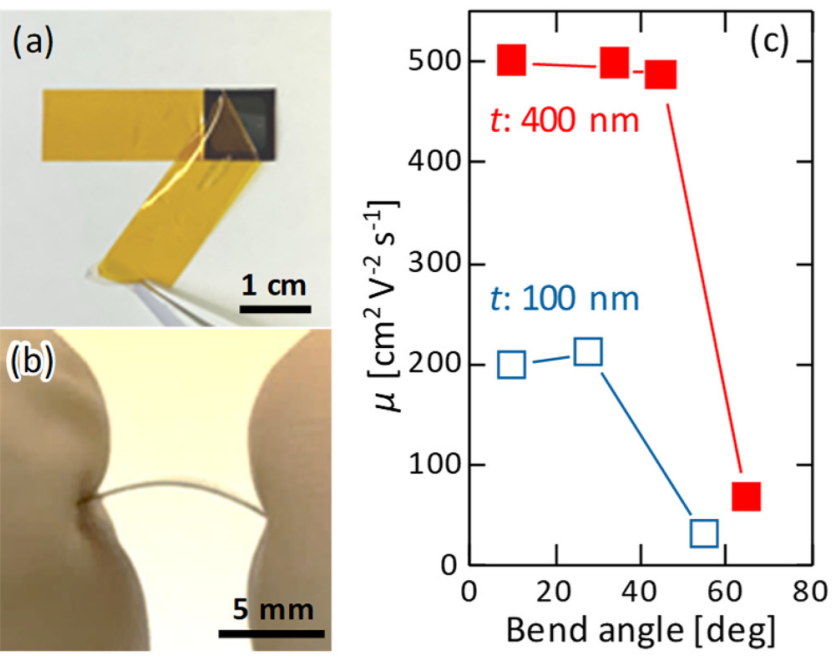

FIG. 6. Mechanical properties of the SPC-Ge layers on a plastic substrate. Photographs showing (a) tape test and (b) bending test for the $t=400 \mathrm{~nm}$ sample. (c) Hole mobility $\mu$ as a function of bend angle for $t=100 \mathrm{~nm}$ and $400 \mathrm{~nm}$, where the samples were measured after being bent at each angle and then returned straight.

Figure 7 shows that the current SPC-Ge layer has the highest $\mu$ among Ge layers formed below the heat-resistant temperature of plastics. This is due to the densification of the a-Ge precursor and the preparation of the $\mathrm{GeO}_{2}$ underlayer. The $\mu$ value is even higher than that for single-crystal Ge layers epitaxially grown from Si-on-insulator substrates ${ }^{10,17}$ and single-crystal Si wafers. These

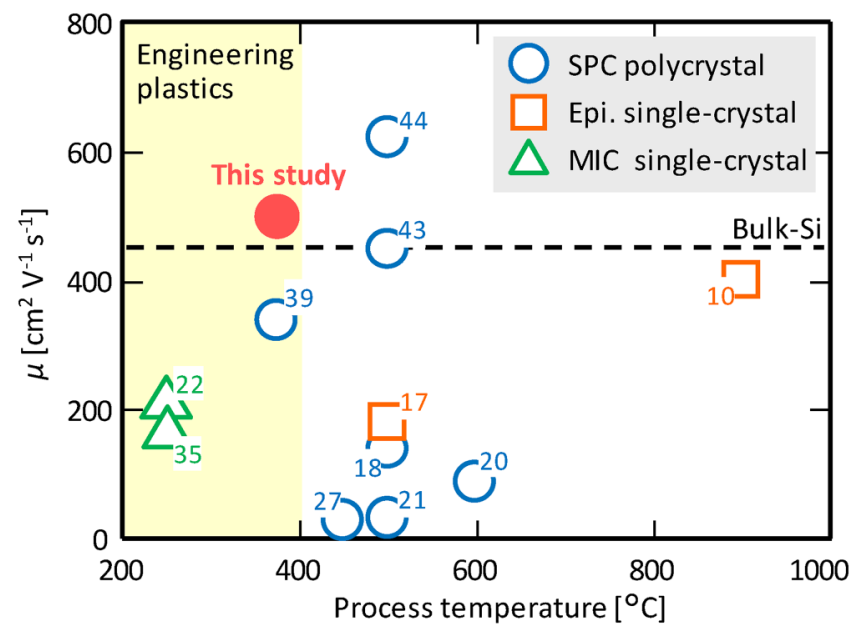

FIG. 7. Comparison of the hole mobility $\mu$ and the process temperature of nondoped Ge layers on insulators with different growth methods, where the data contain various $t$. The reference number is shown near each symbol. The data for single-crystal Si are shown by the dotted line. results mean that single-crystal wafers are no longer necessary for fabricating semiconductor films with a high carrier mobility.

\section{CONCLUSION}

We fabricated SPC-Ge thin films on a $\mathrm{GeO}_{2}$-coated plastic substrate and evaluated their characteristics by comparing with the conventional glass substrate sample. The optical evaluation using XRD and Raman spectroscopies reveal that the plastic sample has a compressive strain while the glass sample has a tensile strain due to the difference in the thermal expansion coefficients between Ge and the substrate. EBSD analyses show that the Ge on plastic has large grains up to $10 \mu \mathrm{m}$, while many intragranular GBs are present. $E_{\mathrm{B}}$ is lower for the plastic sample than for the glass sample, which is discussed in terms of the strain direction. $\mu$ at RT is highly dependent on $t$; a lower $t$ exhibits a lower $\mu$ likely due to high $E_{\mathrm{B}}$ near the $\mathrm{Ge} / \mathrm{GeO}_{2}$ interface. The $\mathrm{Ge}$ layer on plastic with $t=400 \mathrm{~nm}$ exhibits a minimum $E_{\mathrm{B}}(1.3 \mathrm{meV})$ and a maximum $\mu$ $\left(500 \mathrm{~cm}^{2} / \mathrm{V} \mathrm{s}\right) . \mu$ is the highest for a semiconductor thin film synthesized on a plastic substrate, surpassing that of a single-crystal Si wafer. Therefore, we found the strain effects on poly-Ge and demonstrate a high carrier mobility on a plastic substrate. These achievements will lead to high-performance, lightweight, economical, and flexible electronics with multifunctionality.

\section{ACKNOWLEDGMENTS}

This work was financially supported by a Grants-in-Aid for JSPS Research Fellow (No. 19J21034) and the JST PRESTO (No. JPMJPR17R7). The authors are grateful to Professor T. Sakurai (University of Tsukuba) for his assistance with the Hall effect measurement. Some experiments were conducted at the International Center for Young Scientists in NIMS.

\section{DATA AVAILABILITY}

The data that support the findings of this study are available from the corresponding author upon reasonable request.

\section{REFERENCES}

${ }^{1}$ K. Saraswat, C. O. Chui, T. Krishnamohan, D. Kim, A. Nayfeh, and A. Pethe, Mater. Sci. Eng. B 135, 242 (2006).

${ }^{2}$ D. P. Brunco, B. De Jaeger, G. Eneman, J. Mitard, G. Hellings, A. Satta, V. Terzieva, L. Souriau, F. E. Leys, G. Pourtois, M. Houssa, G. Winderickx, E. Vrancken, S. Sioncke, K. Opsomer, G. Nicholas, M. Caymax, A. Stesmans, J. Van Steenbergen, P. W. Mertens, M. Meuris, and M. M. Heyns, J. Electrochem. Soc. 155, H552 (2008).

${ }^{3}$ R. Pillarisetty, Nature 479, 324 (2011).

${ }^{4}$ K. Yamamoto, T. Sada, D. Wang, and H. Nakashima, Appl. Phys. Lett. 103, 122106 (2013).

${ }^{5_{S}}$. Takagi, R. Zhang, J. Suh, S.-H. Kim, M. Yokoyama, K. Nishi, and M. Takenaka, Jpn. J. Appl. Phys, 54, 06FA01 (2015).

${ }^{6}$ A. Toriumi and T. Nishimura, Jpn. J. Appl. Phys. 57, 010101 (2018).

${ }^{7}$ G. Taraschi, A. J. Pitera, and E. A. Fitzgerald, Solid State Electron. 48, 1297 (2004).

${ }^{8}$ Y. Moriyama, K. Ikeda, Y. Kamimuta, M. Oda, T. Irisawa, Y. Nakamura, A. Sakai, and T. Tezuka, Solid State Electron. 83, 42 (2013).

${ }^{9}$ K. Yu, F. Yang, H. Cong, L. Zhou, Q. Liu, L. Zhang, B. Cheng, C. Xue, Y. Zuo, and C. Li, J. Alloys Compd. 750, 182 (2018). 
${ }^{10}$ T. Maeda, K. Ikeda, S. Nakaharai, T. Tezuka, N. Sugiyama, Y. Moriyama, and S. Takagi, Thin Solid Films 508, 346 (2006).

${ }^{11}$ J. Feng, G. Thareja, M. Kobayashi, S. Chen, A. Poon, Y. Bai, P. B. Griffin, S. S. Wong, Y. Nishi, and J. D. Plummer, IEEE Electron Device Lett. 29, 805 (2008).

${ }^{12}$ S. Hu, P. W. Leu, A. F. Marshall, and P. C. McIntyre, Nat. Nanotechnol. 4, 649 (2009).

${ }^{13}$ K. Toko, Y. Ohta, T. Tanaka, T. Sadoh, and M. Miyao, Appl. Phys. Lett. 99, 032103 (2011).

${ }^{14}$ T. Hosoi, Y. Suzuki, T. Shimura, and H. Watanabe, Appl. Phys. Lett. 105, 173502 (2014).

${ }^{15}$ K. Usuda, Y. Kamata, Y. Kamimuta, T. Mori, M. Koike, and T. Tezuka, Appl. Phys. Express 7, 056501 (2014).

${ }^{16}$ Y. Kamata, M. Koike, E. Kurosawa, M. Kurosawa, H. Ota, O. Nakatsuka, S. Zaima, and T. Tezuka, Appl. Phys. Express 7, 121302 (2014).

${ }^{17}$ O. Nakatsuka, N. Tsutsui, Y. Shimura, S. Takeuchi, A. Sakai, and S. Zaima, Jpn. J. Appl. Phys. 49, 04DA10 (2010).

${ }^{18}$ K. Toko, I. Nakao, T. Sadoh, T. Noguchi, and M. Miyao, Solid State Electron. 53, 1159 (2009).

${ }^{19}$ M. Tada, J.-H. Park, D. Kuzum, G. Thareja, J. R. Jain, Y. Nishi, and K. C. Saraswat, J. Electrochem. Soc. 157, H371 (2010).

${ }^{20}$ C.-Y. Tsao, J. Huang, X. Hao, P. Campbell, and M. A. Green, Sol. Energy Mater. Sol. Cells 95, 981 (2011).

${ }^{21}$ H.-W. Jung, W.-S. Jung, H.-Y. Yu, and J.-H. Park, J. Alloys Compd. 561, 231 (2013).

${ }^{22}$ J.-H. Park, K. Kasahara, K. Hamaya, M. Miyao, and T. Sadoh, Appl. Phys. Lett. 104, 252110 (2014).

${ }^{23}$ M. Kurosawa, N. Taoka, H. Ikenoue, O. Nakatsuka, and S. Zaima, Appl. Phys. Lett. 104, 061901 (2014).

${ }^{24}$ K. Toko, R. Numata, N. Oya, N. Fukata, N. Usami, and T. Suemasu, Appl. Phys. Lett. 104, 022106 (2014).

${ }^{25}$ Z. Wang, L. P. H. Jeurgens, W. Sigle, and E. J. Mittemeijer, Phys. Rev. Lett. 115, 016102 (2015).

${ }^{\mathbf{2 6}}$ R. Matsumura, H. Chikita, Y. Kai, T. Sadoh, H. Ikenoue, and M. Miyao, Appl. Phys. Lett. 107, 262106 (2015).

${ }^{27}$ W. Takeuchi, N. Taoka, M. Kurosawa, M. Sakashita, O. Nakatsuka, and S. Zaima, Appl. Phys. Lett. 107, 022103 (2015).

${ }^{28}$ C. Xu, X. Gong, M. Miyao, and T. Sadoh, Appl. Phys. Lett. 115, 042101 (2019).

${ }^{\mathbf{2 9}}$ T. Sadoh, H. Kamizuru, A. Kenjo, and M. Miyao, Appl. Phys. Lett. 89, 192114 (2006).
${ }^{30}$ S. Kabuyanagi, T. Nishimura, K. Nagashio, and A. Toriumi, Thin Solid Films 557, 334 (2014).

${ }^{31}$ A. Hara, Y. Nishimura, and H. Ohsawa, Jpn. J. Appl. Phys. 56, $03 B B 01$ (2017).

${ }^{32}$ H. A. Kasirajan, W.-H. Huang, M.-H. Kao, H.-H. Wang, J.-M. Shieh, F.-M. Pan, and C.-H. Shen, Appl. Phys. Express 11, 101305 (2018).

${ }^{33}$ M. Asadirad, Y. Gao, P. Dutta, S. Shervin, S. Sun, S. Ravipati, S. H. Kim, Y. Yao, K. H. Lee, A. P. Litvinchuk, V. Selvamanickam, and J.-H. Ryou, Adv. Electron. Mater. 2, 1600041 (2016).

${ }^{34}$ B. Hekmatshoar, S. Mohajerzadeh, D. Shahrjerdi, and M. D. Robertson, Appl. Phys. Lett. 85, 1054 (2004).

${ }^{35}$ K. Kasahara, Y. Nagatomi, K. Yamamoto, H. Higashi, M. Nakano, S. Yamada, D. Wang, H. Nakashima, and K. Hamaya, Appl. Phys. Lett. 107, 142102 (2015).

${ }^{36}$ T. Suzuki, B. M. Joseph, M. Fukai, M. Kamiko, and K. Kyuno, Appl. Phys. Express 10, 095502 (2017).

${ }^{37}$ H. Higashi, K. Kudo, K. Yamamoto, S. Yamada, T. Kanashima, I. Tsunoda, H. Nakashima, and K. Hamaya, J. Appl. Phys. 123, 215704 (2018).

${ }^{38}$ H. Haesslein, R. Sielemann, and C. Zistl, Phys. Rev. Lett. 80, 2626 (1998).

${ }^{39} \mathrm{~K}$. Toko, R. Yoshimine, K. Moto, and T. Suemasu, Sci. Rep. 7, 16981 (2017).

${ }^{40}$ D. Takahara, K. Moto, T. Imajo, T. Suemasu, and K. Toko, Appl. Phys. Lett. 114, 082105 (2019).

${ }^{41}$ M. Saito, K. Moto, T. Nishida, T. Suemasu, and K. Toko, Sci. Rep. 9, 16558 (2019).

${ }^{42}$ K. Moto, K. Yamamoto, T. Imajo, T. Suemasu, H. Nakashima, and K. Toko, Appl. Phys. Lett. 114, 212107 (2019).

${ }^{43}$ R. Yoshimine, K. Moto, T. Suemasu, and K. Toko, Appl. Phys. Express 11, $031302(2018)$

${ }^{44}$ T. Imajo, K. Moto, R. Yoshimine, T. Suemasu, and K. Toko, Appl. Phys. Express 12, 015508 (2019).

${ }^{45}$ H. Chen, Y. K. Li, C. S. Peng, H. F. Liu, Y. L. Liu, Q. Huang, J. M. Zhou, and Q.-K. Xue, Phys. Rev. B 65, 233303 (2002)

${ }^{46}$ S. A. Lyon, R. J. Nemanich, N. M. Johnson, and D. K. Biegelsen, Appl. Phys. Lett. 40, 316 (1982).

${ }^{47}$ P. Lengsfeld, N. H. Nickel, C. Genzel, and W. Fuhs, J. Appl. Phys. 91, 9128 (2002).

${ }^{48}$ S. Wolf and R. N. Taubner, Silicon Processing for the VLSI Era: Process Technology (Lattice, Sunset Beach, CA, 1986), Vol. I.

${ }^{49}$ K. Toko, H. Kanno, A. Kenjo, T. Sadoh, T. Asano, and M. Miyao, Appl. Phys. Lett. 91, 042111 (2007).

${ }^{50}$ K. Toko, T. Sadoh, and M. Miyao, Appl. Phys. Lett. 94, 192106 (2009).

${ }^{51}$ J. Y. W. Seto, J. Appl. Phys. 46, 5247 (1975). 